\title{
Functional diversification of IgGs through Fc glycosylation
}

\author{
Taia T. Wang ${ }^{1,2}$ and Jeffrey V. Ravetch ${ }^{3}$ \\ 'Department of Medicine, Division of Infectious Diseases, and Department of Microbiology and Immunology, Stanford University School of Medicine, Stanford, California, USA. ${ }^{2}$ Chan Zuckerberg Biohub, \\ San Francisco, California, USA. ${ }^{3}$ Laboratory of Molecular Cenetics and Immunology, The Rockefeller University, New York, New York, USA.
}

\begin{abstract}
IgC antibodies are secreted from B cells and bind to a variety of pathogens to control infections as well as contribute to inflammatory diseases. Many of the functions of IgGs are mediated through Fc $\gamma$ receptors (FcyRs), which transduce interactions with immune complexes, leading to a variety of cellular outcomes depending on the Fc $\gamma$ Rs and cell types engaged. Which FcyRs and cell types will be engaged during an immune response depends on the structure of Fc domains within immune complexes that are formed when IgGs bind to cognate antigen(s). Recent studies have revealed an unexpected degree of structural variability in IgC Fc domains among people, driven primarily by differences in IgC subclasses and $\mathrm{N}$-linked glycosylation of the $\mathrm{CH} 2$ domain. This translates, in turn, to functional immune diversification through type I and type II FcyR-mediated cellular functions. For example, Fc domain sialylation triggers conformational changes of IgG1 that enable interactions with type II Fc $\gamma \mathrm{Rs}$; these receptors mediate cellular functions including antiinflammatory activity or definition of thresholds for B cell selection based on B cell receptor affinity. Similarly, presence or absence of a core fucose alters type I Fc $\mathrm{R}$ binding of IgC1 by modulating the Fc's affinity for FcyRIIla, thereby altering its proinflammatory activity. How heterogeneity in IgC Fc domains contributes to human immune diversity is now being elucidated, including impacts on vaccine responses and susceptibility to disease and its sequelae during infections. Here, we discuss how Fc structures arising from sialylation and fucosylation impact immunity, focusing on responses to vaccination and infection. We also review work defining individual differences in Fc glycosylation, regulation of Fc glycosylation, and clinical implications of these pathways.
\end{abstract}

\section{Introduction}

IgG antibodies act as a bridge between the host and foreign antigens, coupling antigen detection with the recruitment of innate and adaptive immune processes. This capacity arises from the presence of two functional domains: the antigen-binding Fab domain and the Fc domain, which interacts with Fc $\gamma$ receptors (Fc $\gamma$ Rs) to mediate an array of cellular effector functions $(1,2)$. Diversification of IgG-mediated effector functions is achieved by structural variation in Fc domains; Fc domain structure determines the Fc $\gamma$ Rs, and in turn the effector cells, that can be engaged. One critical determinant of Fc structure that can impact both adaptive and innate Fc $\gamma \mathrm{R}$ signaling pathways is glycosylation of the Fc. In this Review, we will discuss how Fc sialylation and fucosylation impact the functionality of IgG1 antibodies as well as existing and potential clinical applications for IgGs with specific glycan modifications.

The activity of $\operatorname{IgG}$ antibodies depends on both their IgG subclass and Fc glycosylations (3-6). IgGs are found in four subclasses (IgG1-4) in humans, with IgG1 and IgG3 having the highest affinity for activating type I Fc $\gamma$ Rs. Aside from subclass, Fc structure is fur-

Conflict of interest: TTW is a named inventor on a patent $(10,300,127)$ and patent applications relating to IgG Fc effector functions and glycan modifications. JVR is a consultant or member of the scientific advisory board of Harpoon, Kleo, Momenta, Palleon, Portola, Vir, and Xencor and receives consulting fees and stock options. He also has stock holdings in Macrogenics. He is a named inventor on patent numbers: 10,300,127; 10,167,332; 9,845,358; 9,657,101; 9,587,025; 9,481,724; 9,134,310; 8,815,237; $8,618,251 ; 8,470,318 ; 7,846,744$; and $7,416,726$ and on patent applications relating to Fc modifications.

Copyright: () 2019, American Society for Clinical Investigation.

Reference information: J Clin Invest. 2019;129(9):3492-3498.

https://doi.org/10.1172/JCI130029. ther defined by the precise composition of a complex, biantennary $\mathrm{N}$-linked glycan present at Asp297 of each CH2 domain (Figure 1, upper left). A core glycan is always present, composed of seven saccharide units: four $\mathrm{N}$-acetylglucosamine and three mannose residues. Removal of the core glycan diminishes affinity of the Fc for Fc $\gamma$ Rs, translating to loss of Fc $\gamma$ R-mediated effector functions in vivo (7-13). Despite the requirement of Fc glycosylation for $\mathrm{Fc}-\mathrm{Fc} \gamma \mathrm{R}$ interactions, the molecular interactions between the Fc and $\mathrm{F} c \gamma \mathrm{Rs}$ are mediated primarily by amino acid residues rather than saccharide residues (14). One exception to this is afucosylated Fc glycoforms, which stabilize interactions with Fc $\gamma$ RIIIa and Fc $\gamma$ RIIIb via a sugar-based interaction (15). Generally, the role of the Fc glycan is in regulating structure of the $\mathrm{Fc}$ by determining the degree of its conformational stability and thus interactions between the Fc and type I or type II FcyRs (9, 13, 16-18). The ability of the $\mathrm{Fc}$ to adopt distinct structures arises from its organization into two chains, each composed of two domains, termed $\mathrm{CH} 2$ and $\mathrm{CH} 3$. The two chains of the IgG heavy chain that make up the $\mathrm{Fc}$ are bound by disulfide bonds in the hinge-proximal $\mathrm{CH} 2$ region without extensive protein-protein interactions between the $\mathrm{CH} 3$ domains, resulting in a horseshoe-like configuration for the dimeric structure. Absence of additional bonds between the $\mathrm{CH} 2$ or $\mathrm{CH} 3$ domains of the $\mathrm{Fc}$ results in its capacity for conformational flexibility.

The core Fc glycan can be modified by specific saccharide units, including core fucosylation, bisecting GlcNAcylation, and galactosylation at one or both arms; the presence of galactose enables addition of terminal sialic acid(s). The terminal sialic acid modification, in the presence of core fucose, destabilizes the IgG1 Fc, enabling sampling of a "closed" Fc conformation and associ- 


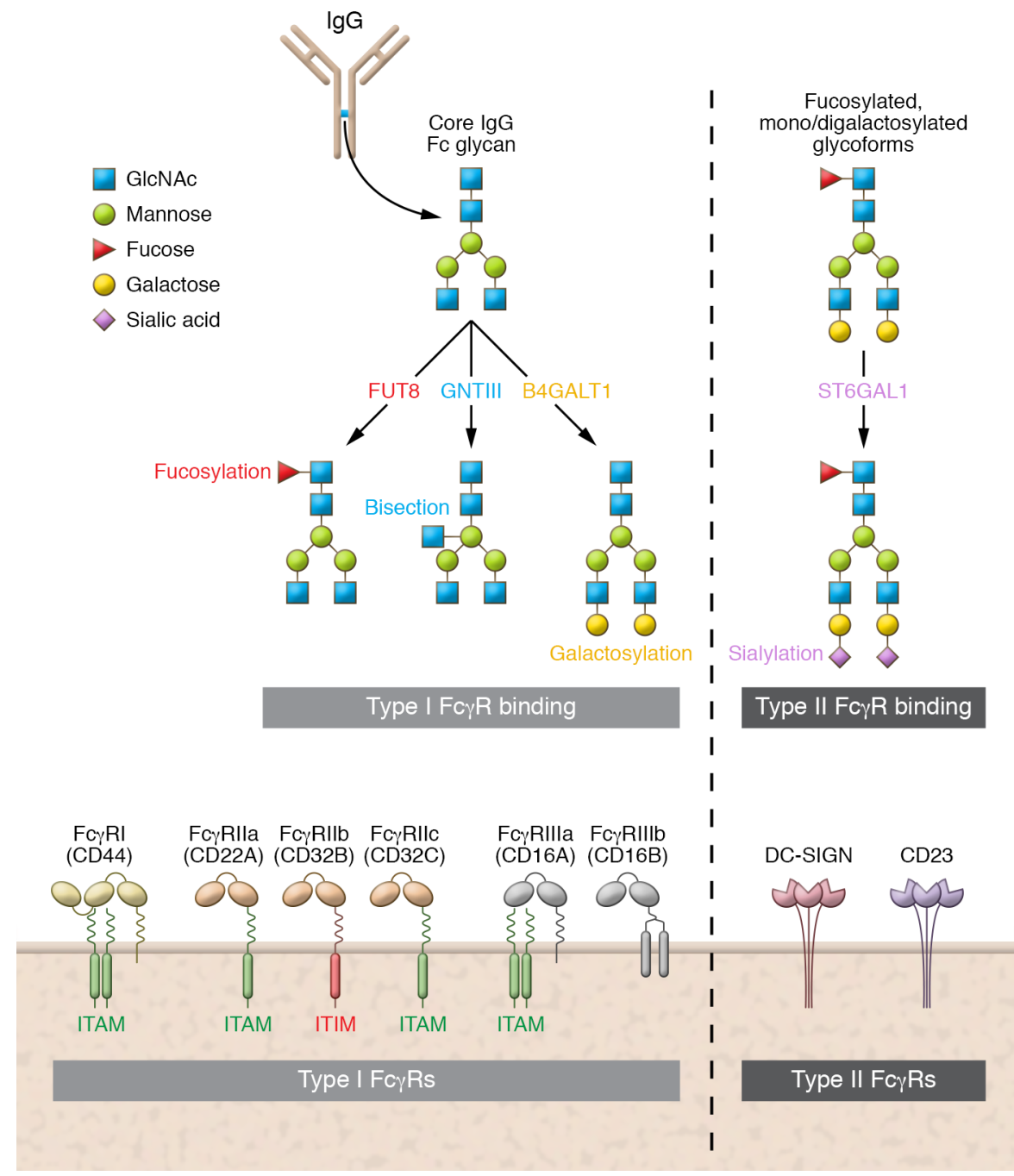

Figure 1. The core IgG Fc glycan modifications and type I and type II Fc $\gamma$ Rs. Top: The core Fc glycan is attached within the $\mathrm{CH} 2$ domain of each IgG heavy chain and can be modified by various glycosyltransferases for addition of fucose (FUT8), galactose (B4GALT1), $N$-acetylglucosamine (GNTIII), and sialic acid (ST6GAL1) residues. Although sialylation without fucosylation does not impact the enhanced binding of the afucosylated glycoforms to the type I Fc $\gamma R$ Fc $\gamma$ RIIla, sialylation of fucosylated glycoforms destabilizes the Fc domain, enabling structural rearrangement that favors type II Fc $\gamma \mathrm{R}$ binding. Bottom: Type I Fc $\gamma$ Rs are members of the immunoglobulin super family and transduce activating or inhibitory signaling on the basis of the presence of an intracellular immunoreceptor tyrosine-based activation motif (ITAM) or immunoreceptor tyrosine-based inhibitory motif (ITIM) motif. Type II Fc $\gamma$ Rs are the C-type lectins DC-SICN and CD23, which mediate antiinflammatory activity and B cell modulatory activities, respectively. Type II Fc $\gamma R$ s are distinguished by their ability to engage sialylated, fucosylated immune complexes. ation with type II Fc $\gamma$ Rs (Figure 1, upper right and refs. 9, 17, 18). Absence of core fucose enhances affinity of the Fc for the type I Fc $\gamma$ RIIIa through interactions between the carbohydrate moieties on Fc $\gamma$ RIIIa and the IgG1 Fc (15); this occurs regardless of sialylation status (17). While each of these saccharide modifications likely plays a role in regulating the $\mathrm{Fc}-\mathrm{Fc} \gamma \mathrm{R}$ interactions of various IgG subclasses their activities have been best studied in the context of the IgG1 subclass. As little is known about the impact of sialylation or fucosylation on IgG2-IgG4 at this time, discussions in this Review refer specifically to sialylation and fucosylation of IgG1 antibodies.

\section{Fc $\gamma$ receptors}

Type I and type II Fc $\gamma$ Rs are distinguished by their ability to interact with Fcs based on specific glycan modifications. Type I Fc $\gamma$ Rs (Fc $\gamma$ RI, Fc $\gamma$ RIIa, Fc $\gamma$ RIIb, Fc $\gamma$ RIIc, Fc $\gamma$ RIIIa, Fc $\gamma$ RIIIb) are members of the immunoglobulin superfamily and show reduced binding to sialylated Fcs relative to those without sialic acid modification (19), while the absence of fucose uniquely enhances Fc $\gamma$ RIIIa and Fc $\gamma$ RIIIb binding (Fc $\gamma$ RIIIb is a low-affinity type I Fc $\gamma$ R without a signaling domain) (20). Recruitment of effector cells through Fctype I Fc $\gamma \mathrm{R}$ interactions is required for a major subset of antibodymediated functions, including those involved in protection against many infectious diseases and in tumor immunotherapy (21-28). Type II Fc $\gamma$ Rs are more recently described and include human dendritic cell-specific ICAM3-grabbing non-integrin (DC-SIGN/ CD209) and CD23 (FceRII), both C-type lectin receptors that can be engaged by sialylated Fc domains within immune complexes (Figure 1, bottom and refs. 9, 18, 29, 30). Type II Fc $\gamma$ Rs mediate distinct modulatory activities including antiinflammatory signaling (DC-SIGN) (30-32) and modification of selection of B cells in the germinal center (CD23) (29), and likely mediate additional activities yet to be defined. In addition to DC-SIGN and CD23, sialylated Fcs may interact with other C-type lectin receptors such as CD22, CLEC4M, CLEC4G, and CLEC4A (DCIR), but the biological significance of those interactions is not yet known (33-35).

DC-SIGN is expressed on dendritic cells and on subsets of monocytes/macrophages and B cells (36-38). While DC-SIGN can act as an Fc $\gamma \mathrm{R}$, it can also engage other ligands, including a variety of pathogen-associated glycoproteins (39-43). Binding of DC-SIGN to these ligands occurs predominantly through carbohydrate-mediated interactions. This contrasts with the mechanism of sialylated immune complex-DC-SIGN interactions, which occur through protein-based interactions in a region of the $\mathrm{Fc}$ that becomes exposed upon sialylation $(9,18,44)$. 


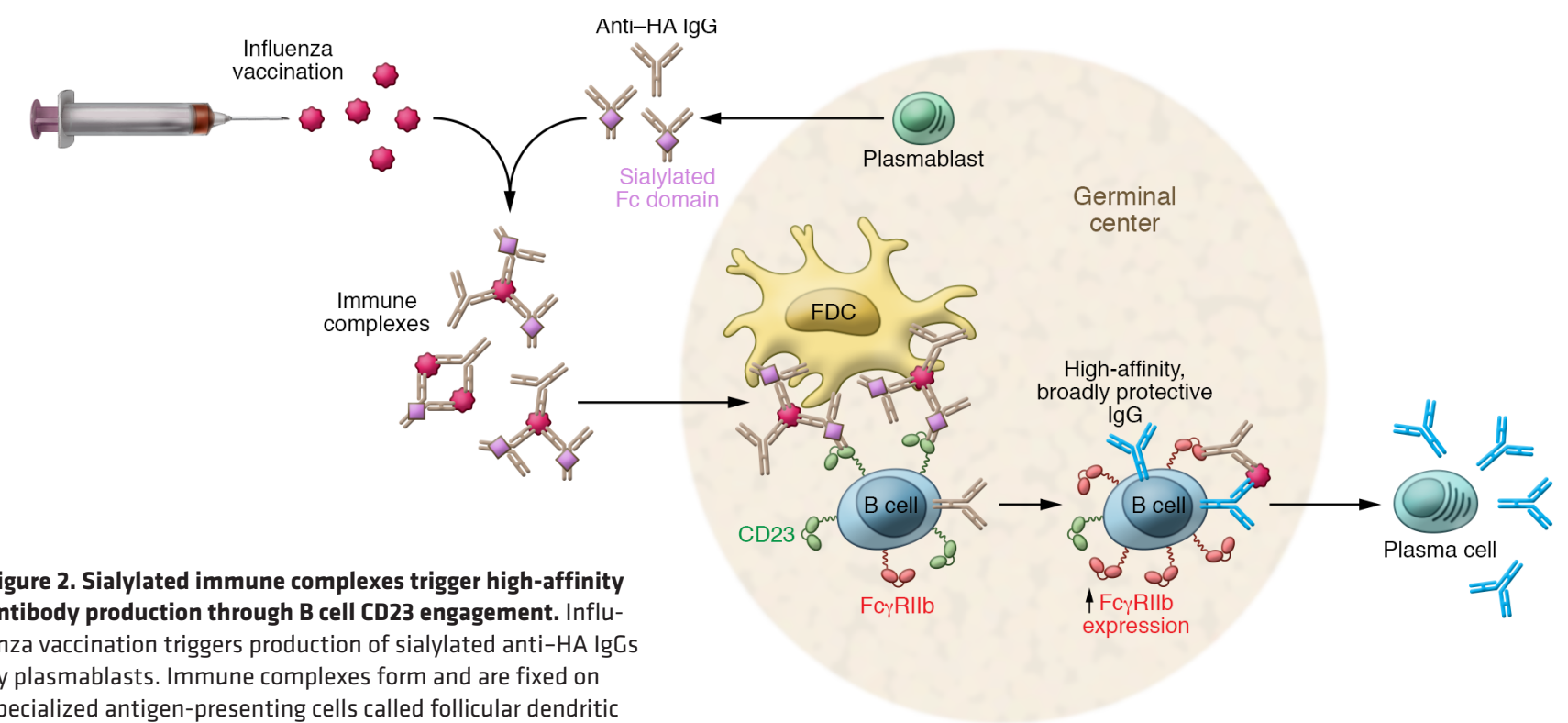
specialized antigen-presenting cells called follicular dendritic cells (FDCs) within the germinal center. Coengagement of CD23 with the B cell receptor by sialylated $\mathrm{HA}$ immune complexes induces increased expression of the inhibitory Fc $\gamma$ RIlb, resulting in selection of higher-affinity B cells.

CD23 exists as two splice variants, CD23a and CD23b, which vary by cellular expression and the ability to be induced by IL-4 (45). CD23a is constitutively expressed on B cells, while CD23b expression is induced by IL-4 and is limited to myeloid cells and some $\mathrm{T}$ cell subsets (45). CD23 was originally described as the low-affinity receptor for $\operatorname{IgE}$ and has been proposed to function in regulation of IgE synthesis (46). The ability of CD23 to function as both an IgE and IgG Fc receptor was first suggested by modeling studies showing a high degree of structural homology between the proposed IgE-CD23 and sialylated IgG Fc-CD23 complexes $(9,47$, 48). Subsequent in vitro and in vivo studies confirmed that CD23 can act as a receptor for $\operatorname{IgG}(9,29)$. As with DC-SIGN, sialylated immune complex-CD23 interactions are thought to occur through a mechanism of protein-based interactions that depend on flexibility of the IgG CH2 domain and conformational changes that occur upon Fc sialylation $(9,18)$.

With the exception of Fc $\gamma$ RI, other type I and type II Fc $\gamma$ Rs have low affinity for Fcs such that Fc-Fc $\mathrm{R}$ interactions take place primarily in the context of immune complex formation, when avidity-based interactions can occur. This requisite immune complex formation confers specificity to the effector cell response, but which Fc $\gamma$ Rs and effector cells can be engaged by a given immune complex is determined by the structure of Fc domains in the complex. For example, immune complexes that are rich in IgG1 and IgG3 subclasses will recruit activating cellular functions over those rich In IgG2; immune complexes rich in fucose and sialic acid will mediate modulatory/antiinflammatory activities relative to those with reduced fucose and sialic acids. Thus, the specific distribution of IgG subclasses and posttranslational modifications of Fcs that is produced by an individual will determine the quality of antibodies produced by vaccination or after infection and is likely a central determinant of pathogenicity of autoantibodies.

\section{Inflammation}

Balanced signaling through activating and inhibitory Fc $\gamma$ Rs is required for inflammatory responses that contribute to health rather than to disease. A central mechanism for regulation of inflammatory responses is structural diversification of the IgG1 Fc via glycosylation. Fucosylation and sialylation are the two primary Fc modifications that impact recruitment of inflammatory effector cell responses. Reduced core fucosylation or reduced sialylation of the Fc promotes increased activating Fc $\gamma \mathrm{R}$ signaling and, in turn, enhances inflammatory effector cell activity. Core fucosylation regulates interactions with the activating type I Fc $\gamma$ R, Fc $\gamma$ RIIIa, with absence of a core fucose moiety resulting in stabilization of the Fc with Fc $\gamma$ RIIIa and enhanced signaling. In contrast, Fc sialylation is destabilizing, leading to sampling of the "closed" Fc conformation, which diminishes type I Fc $\gamma$ R interactions and enables interactions between the Fc and type II Fc $\gamma$ Rs (Figure 1 and refs. 9, 18, 19, 29-31).

Destabilization of the Fc upon sialylation leading to the "closed" Fc conformation was originally demonstrated through biochemical methods and by crystallization studies $(9,18,19)$. While the dependence of effector functions mediated by sialylated Fcs on type II Fc $\gamma$ Rs is readily demonstrated in vivo, interactions between sialylated Fcs and type II Fc $\gamma$ Rs in vitro can be more sensitive to specific assay conditions than are Fc-type I Fc $\gamma$ R interactions. This may be due to alternation between the "open" and "closed" Fc conformations upon sialylation, resulting in reduced stabilization and affinity for type II Fc $\gamma$ Rs. The dependence of activities conferred through sialylated Fcs on type II Fc $\gamma$ Rs has been confirmed in numerous studies using mice that do not express DC-SIGN/CD23. In the case of DC-SIGN, transgenic expression of the human DC-SIGN receptor in the SIGN-R1 (murine DC-SIGN ortholog) knockout mouse model rescues the biological activity associated with sialylated Fc administration $(29,30,44,49)$. 
Shifts in signaling through different type I and type II Fc $\gamma$ Rs can have considerable clinical consequences. For example, increased activating type I Fc $\gamma \mathrm{R}$ signaling due to reduced fucosylation of $\operatorname{IgG}$ is associated with progression to severe disease in secondary dengue infections (50) and has been implicated in neonatal alloantibody-mediated disease (51-53). Promoting type I Fc $\gamma \mathrm{R}$ signaling can also have pronounced therapeutic effects as observed in the context of antibodies that mediate broad immunity against influenza viruses $(25,54,55)$. Another important example is the improved protective activity that can be achieved by engineering of tumor immunotherapies toward activating type I Fc $\gamma$ Rs. For example, outcomes of anti-CD20 immunotherapy in chronic lymphocytic leukemia patients are improved by glycoengineering of the antibody to engage the activating Fc $\gamma$ RIIIa with higher affinity (56). Further, recent clinical studies found that progression-free survival after anti-HER2 immunotherapy for metastatic breast cancer is significantly improved by engineering of the anti-HER $2 \mathrm{mAb}$ for enhanced activating type I Fc $\gamma \mathrm{R}$ binding affinity and reduced affinity for the inhibitory Fc $\gamma$ RIIb (57). This improved survival after administration of the Fc-optimized anti-HER2 $\mathrm{mAb}$ may involve a vaccinal response whereby administration of an anti-tumor $\mathrm{mAb}$ mediates processing and presentation of the mAb's target protein, promoting maturation of $\mathrm{T}$ cells against the target, which can provide long-term protection against tumor growth (58).

Shifts in Fc $\gamma \mathrm{R}$ binding that increase type II Fc $\gamma \mathrm{R}$-mediated activities occur in the presence of IgGs with sialylated Fcs. This can result in antiinflammatory activity, as observed with administration of high-dose intravenous immunoglobulin (IVIg). IVIg is purified IgG, pooled from thousands of donors, and has long been observed to mediate therapeutic activity in the setting of acute inflammatory diseases such as immune thrombocytopenia and chronic inflammatory demyelinating polyneuropathy. The antiinflammatory activity in IVIg arises from the fraction of IgGs within the pool that are modified by sialylated Fc glycoforms (6). Mechanisms involved in antiinflammatory activity of sialylated IgGs have been dissected in studies using mice expressing humanized DC-SIGN, the type II Fc $\gamma \mathrm{R}$ required for antiinflammatory activity of IVIg. These studies showed that DC-SIGN can transduce antiinflammatory activity by inducing production of IL-33 upon engagement by sialylated Fc IgGs. IL-33 production, in turn, led to release of IL- 4 by basophils, which ultimately induced increased expression of Fc $\gamma$ RIIb on effector myeloid cells (30). Expression of the inhibitory Fc $\gamma$ RIIb modulates the threshold of activation of inflammatory cells, with increased Fc $\gamma$ RIIb expression leading to reduced proinflammatory cytokine production (5961). In this way, IVIg administration (or increasing endogenous Fc sialylation) can resolve acute inflammatory responses (31, 32, 49). Studies revealing the mechanistic basis of the antiinflammatory activity of IVIg suggest that endogenous IgG sialylation levels may drive susceptibility to inflammatory diseases.

\section{Adaptive immunity}

Aside from modulating inflammatory responses, Fc glycosylation has a separate function in regulation of adaptive immune responses. Antibodies that complex with vaccine antigens alter the way antigens are processed and can thus modulate the ulti- mate $\mathrm{B}$ and $\mathrm{T}$ cell responses to vaccination. The role of sialylated immune complexes in B cell selection was recently discovered in work drawing on the observation that sialylation of antiinfluenza hemagglutinin IgG1 increases in the days following seasonal influenza vaccination in humans $(29,62)$. This work observed that enhanced sialylation correlated with increased antibody affinity after vaccination, implicating sialylated immune complexes within the germinal center in modulation of B cell selection after vaccination.

Selection of B cells based on affinity of the B cell receptor (BCR) is achieved, in part, by signaling through the inhibitory Fc $\gamma$ RIIb on B cells, which acts to counterbalance the activating signal transduced through the BCR bound by antigen. In the absence of inhibitory Fc $\gamma$ RIIb or with low-level expression or signaling, B cells lack appropriate activation thresholds and produce highertiter, low-avidity IgGs $(63,64)$. Insufficient Fc $\gamma$ RIIb signaling can lead to failure of immune tolerance, with autoantibody production in $\mathrm{Fc}_{\mathrm{RIIb}}{ }^{-/}$mice and low levels of B cell Fc $\gamma \mathrm{RIIb}$ found in patients with autoantibody-mediated diseases (64-71). In contrast, antigens presented in the form of immune complexes within germinal centers can engage the BCR but also Fc $\gamma$ RIIb, thus elevating the threshold of $\mathrm{BCR}$ signaling required for activation of the $\mathrm{B}$ cell, which ultimately drives higher-affinity B cell responses $(68,72-74)$.

The mechanism by which sialylated IgGs impact B cell selection relies, at least in part, on the BCR-Fc $\gamma$ RIIb axis. Sialylated immune complexes drive increased expression of Fc $\gamma$ RIIb on $B$ cells in a CD23-dependent manner. Increased FcyRIIb expression, in turn, elevates the threshold of BCR signaling required for cellular activation (Figure 2). Thus, immunization with sialylated hemagglutinin (HA) immune complexes results in higher-avidity IgG responses over immunization with asialylated HA immune complexes in CD23-expressing mice $(29,75)$. These experiments revealed a molecular mechanism whereby sialylated immune complexes can regulate the affinity of $\mathrm{B}$ cell responses after vaccination and also defined basal antibody sialylation as a driver of heterogeneity in the quality of vaccine responses. An important topic for future studies is whether the CD23 pathway can be targeted to improve the quality of antibody responses elicited additional vaccines. This could be especially useful in settings where avidity of the antibody response is a known determinant of vaccine efficacy, as with polysaccharide-based vaccines.

\section{Heterogeneity and regulation of Fc domain glycosylation}

A fascinating observation that has emerged from studies of human IgG repertoires in recent years is the considerable heterogeneity that exists in Fc glycosylation. Because antibody fucosylation and sialylation are central determinants of inflammatory thresholds, differences in these Fc saccharides among people are likely a key driver of distinctions in basal susceptibilities to inflammatory diseases (76-81).

Basal IgG1 Fc sialylation can differ by approximately $30 \%$ among people; this amount of variation is well within a range that modulates type II Fc $\gamma \mathrm{R}$-mediated effector functions $(29,75$, $81,82)$. IgG1 Fc fucosylation varies by more than $20 \%$ among adults, with a majority of healthy adults having more than $90 \%$ fucosylation. Intriguingly, individuals appear to have set points 
for Fc sialylation and fucosylation. This is observed in vaccination studies in which serial samples are taken over several weeks after vaccination. These studies show that even following large vaccine-elicited changes in Fc glycosylation, there is ultimately a general restoration of the abundance of various glycoforms found on prevaccination IgGs. Thus, people with relatively low sialylation on prevaccination IgGs, for example, can achieve average or high levels during vaccine-induced modulations, but ultimately return to relatively low Fc sialylation $(29,62)$. Why IgG Fc glycosylation differs among individuals is a topic of great interest. With respect to sialylation, recent work has shown that B cell production of the glycosyltransferase ST6GAL1 and sialylated antibody can be modulated by levels of estrogen or by IL-23 in vivo, suggesting that basal distinctions in hormones and cytokines may contribute to diversity in Fc sialylation $(81,83)$. Other general associations have been made between the abundance of different Fc glycoforms and age, sex, pregnancy, and treatment with high-dose IVIg (77, 82, $84,85)$. The cumulative evidence suggests that both heritable and nonheritable factors likely influence set points for Fc sialylation.

Aside from differences in basal Fc sialylation and fucosylation among individuals, another level of heterogeneity is apparent within IgG repertoires of individuals. For example, differences in Fc glycoforms are associated with IgG Fab specificity. The antiHA IgG response is one setting where the abundance of sialylation and fucosylation has been observed to differ depending on specificity of the Fab domain; anti-HA globular head IgGs are significantly more sialylated and fucosylated than are IgGs that react with the HA stem domain (29). In addition to the anti-HA response, other examples of Fc glycosylation segregating on Fab specificity are observed in autoimmune diseases such as granulomatosis with polyangiitis (GPA) and rheumatoid arthritis (RA). In these diseases, anti-PR3 (GPA) and anti-ACPA (RA) are found, and these antibody specificities have reduced $\mathrm{Fc}$ sialylation over total IgG sialylation from the same individuals $(6,78,86)$. That the abundance of IgG Fc sialylation and fucosylation can be linked to antigen or domain specificity may be a consequence of differential glycosylation based on B cell subset. For example, ST6GAL1 and FUT8, the glycosyltransferases responsible for Fc sialylation and fucosylation, respectively, are expressed at higher levels in plasmablasts following influenza vaccination compared with memory or naive B cells (29).

With improved understanding of mechanisms regulating IgG Fc glycosylation, an important goal will be to harness specific pathways for a variety of therapeutic applications. For example, endogenous Fc sialylation could be modulated to treat diseases responsive to IVIg therapy, or vaccination strategies could be designed that would elicit antibodies with optimized Fc fucosylation to improve the effector function of vaccine-elicited antibodies and improve overall vaccine effectiveness.

\section{Future directions}

IgG Fc glycosylation is a mechanism for regulating Fc structure, and as a consequence it also regulates Fc $\gamma \mathrm{R}$ signaling and cellular effector functions. Numerous recent studies have revealed an unexpected degree of diversity in the Fc domain that translates into functional diversification in vivo. These studies have culminated in the understanding that variability among humans in Fc domain structural repertoires is likely a central driver of heterogeneity in human immunity $(6,29,50-53,56,62,78,79,81,87)$. Rather than a static, monomorphic domain, the IgG Fc has evolved the capacity to modulate its structure and thus its binding interactions with families of Fc receptors, conferring a diversity of functional responses that can be associated with unique antigen-binding domains. While the role of sialylation and fucosylation in modulating Fc domain structure is now appreciated, there are considerable gaps in our knowledge about the mechanisms that drive $\mathrm{Fc}$ diversification and the contributions of Fc glycan modifications to the activity of non-IgG1 subclasses. Further, all IgG subclasses are differentially glycosylated among humans, and the consequences of this are largely unknown.

In addition to the effect of Fc glycoforms on non-IgG1 subclasses, an area where basic discovery studies are needed is in definition of receptors required for $\operatorname{IgG}$ function. There almost certainly exist Fc $\gamma$ Rs and cellular activities integral to IgG effector responses that remain to be identified. Vaccination is yet another critical topic for discovery. As effectiveness of vaccine responses generally depends on the Fab repertoire and the effector capacity of vaccine-elicited IgGs, the development of methods to elicit IgGs with specific $\mathrm{Fc}$ domain repertoires during vaccination could be transformative for the prevention and treatment of infectious diseases and could substantially advance antitumor vaccination strategies. Future studies will undoubtedly continue to shed light on how Fc domain repertoires impact susceptibility to human diseases and how Fc $\gamma$ R pathways can be harnessed for therapeutic purposes.

\section{Acknowledgments}

Support was received from Stanford University, the Chan Zuckerberg Biohub, and the Searle Scholars Program. Research reported in this publication was supported in part by the Bill \& Melinda Gates Foundation (OPP1188461) and the NIH (1R01AI13911901A1 and 2U19AI111825-06). Additional support was received from The Rockefeller University and grant UL1TR001866 from the National Center for Advancing Translational Sciences, NIH, and the Clinical and Translational Science Award program.

Address correspondence to: Jeffrey V. Ravetch, Laboratory of Molecular Genetics and Immunology, The Rockefeller University, 1230 York Avenue, New York, New York 10065, USA. Email: ravetch@rockefeller.edu.
1. Bournazos S, Wang TT, Dahan R, Maamary J, Ravetch JV. Signaling by antibodies: recent progress. Annu Rev Immunol. 2017;35:285-311.

2. Pincetic A, et al. Type I and type II Fc receptors regulate innate and adaptive immunity. Nat Immunol. 2014;15(8):707-716.

3. Tao MH, Morrison SL. Studies of aglycosylated chimeric mouse-human IgG. Role of carbohydrate in the structure and effector functions mediated by the human IgG constant region. J Immunol. 1989;143(8):2595-2601.

4. Sarmay G, Lund J, Rozsnyay Z, Gergely J, Jefferis R Mapping and comparison of the interaction sites on the Fc region of IgG responsible for triggering antibody dependent cellular cytotoxicity (ADCC) through different types of human Fc gamma receptor. Mol Immunol. 1992;29(5):633-639.

5. Nimmerjahn F, Ravetch JV. Divergent immunoglobulin g subclass activity through selective Fc receptor binding. Science. 2005;310(5753):1510-1512. 
6. Kaneko Y, Nimmerjahn F, Ravetch JV. Anti-inflammatory activity of immunoglobulin $\mathrm{G}$ resulting from $\mathrm{Fc}$ sialylation. Science. 2006;313(5787):670-673.

7. Borrok MJ, Jung ST, Kang TH, Monzingo AF, Georgiou G. Revisiting the role of glycosylation in the structure of human IgG Fc. ACS Chem Biol. 2012;7(9):1596-1602.

8. Subedi GP, Barb AW. The structural role of antibody $\mathrm{N}$-glycosylation in receptor interactions. Structure. 2015;23(9):1573-1583.

9. Sondermann P, Pincetic A, Maamary J, Lammens K, Ravetch JV. General mechanism for modulating immunoglobulin effector function. Proc Natl Acad Sci U S A. 2013;110(24):9868-9872.

10. Shields RL, et al. High resolution mapping of the binding site on human IgG1 for Fc gamma RI, Fc gamma RII, Fc gamma RIII, and FcRn and design of IgG1 variants with improved binding to the $\mathrm{Fc}$ gamma R. J Biol Chem. 2001;276(9):6591-6604.

11. Walker MR, Lund J, Thompson KM, Jefferis R. Aglycosylation of human IgG1 and IgG3 monoclonal antibodies can eliminate recognition by human cells expressing Fc gamma RI and/or Fc gamma RII receptors. Biochem J. 1989;259(2):347-353.

12. Smith P, DiLillo DJ, Bournazos S, Li F, Ravetch JV. Mouse model recapitulating human $\mathrm{Fc} \gamma$ receptor structural and functional diversity. Proc Natl Acad Sci U S A. 2012;109(16):6181-6186.

13. Mimura $Y$, et al. Role of oligosaccharide residues of IgG1-Fc in Fc gamma RIIb binding. J Biol Chem. 2001;276(49):45539-45547.

14. Sondermann P, Huber R, Oosthuizen V, Jacob U. The 3.2-A crystal structure of the human IgG1 Fc fragment-Fc gammaRIII complex. Nature. 2000;406(6793):267-273.

15. Ferrara C, et al. Unique carbohydratecarbohydrate interactions are required for high affinity binding between FcgammaRIII and antibodies lacking core fucose. Proc Natl Acad Sci US A. 2011;108(31):12669-12674.

16. Mimura Y, et al. The influence of glycosylation on the thermal stability and effector function expression of human IgG1-Fc: properties of a series of truncated glycoforms. Mol Immunol. 2000;37(12-13):697-706.

17. Li T, DiLillo DJ, Bournazos S, Giddens JP, Ravetch JV, Wang LX. Modulating IgG effector function by Fc glycan engineering. Proc Natl Acad Sci U S A. 2017;114(13):3485-3490.

18. Ahmed AA, et al. Structural characterization of anti-inflammatory immunoglobulin $\mathrm{G}$ Fc proteins. JMol Biol. 2014;426(18):3166-3179.

19. Scallon BJ, Tam SH, McCarthy SG, Cai AN, Raju TS. Higher levels of sialylated Fc glycans in immunoglobulin $\mathrm{G}$ molecules can adversely impact functionality. Mol Immunol. 2007;44(7):1524-1534.

20. Liu SD, Chalouni C, Young JC, Junttila TT, Sliwkowski MX, Lowe JB. Afucosylated antibodies increase activation of Fc $\gamma$ RIIIa-dependent signaling components to intensify processes promoting ADCC. Cancer Immunol Res. 2015;3(2):173-183

21. Bournazos S, Chow SK, Abboud N, Casadevall A, Ravetch JV. Human IgG Fc domain engineering enhances antitoxin neutralizing antibody activity. J Clin Invest. 2014;124(2):725-729.

22. Bournazos S, Gazumyan A, Seaman MS,
Nussenzweig MC, Ravetch JV. Bispecific AntiHIV-1 antibodies with enhanced breadth and potency. Cell. 2016;165(7):1609-1620.

23. Clynes R, Takechi Y, Moroi Y, Houghton A, Ravetch JV. Fc receptors are required in passive and active immunity to melanoma. Proc Natl Acad Sci U S A. 1998;95(2):652-656.

24. Clynes RA, Towers TL, Presta LG, Ravetch JV. Inhibitory Fc receptors modulate in vivo cytotoxicity against tumor targets. Nat Med. 2000;6(4):443-446.

25. DiLillo DJ, Palese P, Wilson PC, Ravetch JV. Broadly neutralizing anti-influenza antibodies require Fc receptor engagement for in vivo protection. J Clin Invest. 2016;126(2):605-610.

26. Li F, Ravetch JV. Inhibitory Fc $\gamma$ receptor engagement drives adjuvant and anti-tumor activities of agonistic CD40 antibodies. Science. 2011;333(6045):1030-1034.

27. Zhang $M$, et al. Effective therapy for a murine model of human anaplastic large-cell lymphoma with the anti-CD30 monoclonal antibody, HeFi-1, does not require activating Fc receptors. Blood. 2006;108(2):705-710.

28. Zhang $\mathrm{M}$, et al. Activating Fc receptors are required for antitumor efficacy of the antibodies directed toward CD25 in a murine model of adult t-cell leukemia. Cancer Res. 2004;64(16):5825-5829.

29. Wang TT, et al. Anti-HA glycoforms drive B cell affinity selection and determine influenza vaccine efficacy. Cell. 2015;162(1):160-169.

30. Anthony RM, Kobayashi T, Wermeling F, Ravetch JV. Intravenous gammaglobulin suppresses inflammation through a novel $\mathrm{T}(\mathrm{H}) 2$ pathway. Nature. 2011;475(7354):110-113.

31. Anthony RM, Nimmerjahn F, Ashline DJ, Reinhold VN, Paulson JC, Ravetch JV. Recapitulation of IVIG anti-inflammatory activity with a recombinant IgG Fc. Science. 2008;320(5874):373-376.

32. Washburn N, et al. Controlled tetra-Fc sialylation of IVIg results in a drug candidate with consistent enhanced anti-inflammatory activity. Proc Natl Acad Sci U S A. 2015;112(11):E1297-E1306.

33. Jellusova J, Nitschke L. Regulation of B cell functions by the sialic acid-binding receptors siglec- $\mathrm{G}$ and CD22. Front Immunol. 2011;2:96.

34. Schwab I, Seeling M, Biburger M, Aschermann S, Nitschke L, Nimmerjahn F. B cells and CD22 are dispensable for the immediate antiinflammatory activity of intravenous immunoglobulins in vivo. Eur J Immunol. 2012;42(12):3302-3309.

35. Böhm S, Kao D, Nimmerjahn F. Sweet and sour: the role of glycosylation for the anti-inflammatory activity of immunoglobulin G. Curr Top Microbiol Immunol. 2014;382:393-417.

36. Rappocciolo G, et al. DC-SIGN on B lymphocytes is required for transmission of HIV-1 to T lymphocytes. PLoS Pathog. 2006;2(7):e70.

37. Tassaneetrithep B, et al. DC-SIGN (CD209) mediates dengue virus infection of human dendritic cells. JExp Med. 2003;197(7):823-829.

38. Conde P, et al. DC-SIGN(+) macrophages control the induction of transplantation tolerance. Immunity. 2015;42(6):1143-1158

39. Geijtenbeek TB, et al. DC-SIGN, a dendritic cell-specific HIV-1-binding protein that enhances trans-infection of T cells. Cell. 2000;100(5):587-597.

40. Alvarez CP, Lasala F, Carrillo J, Muñiz O, Corbi
AL, Delgado R. C-type lectins DC-SIGN and L-SIGN mediate cellular entry by Ebola virus in cis and in trans. JVirol. 2002;76(13):6841-6844.

41. Halary F, et al. Human cytomegalovirus binding to DC-SIGN is required for dendritic cell infection and target cell trans-infection. Immunity. 2002;17(5):653-664.

42. Colmenares M, Puig-Kröger A, Pello OM, Corbí AL, Rivas L. Dendritic cell (DC)-specific intercellular adhesion molecule 3 (ICAM-3)grabbing nonintegrin (DC-SIGN, CD209), a C-type surface lectin in human DCs, is a receptor for Leishmania amastigotes. J Biol Chem. 2002;277(39):36766-36769.

43. Maeda N, et al. The cell surface receptor DC-SIGN discriminates between Mycobacterium species through selective recognition of the mannose caps on lipoarabinomannan. J Biol Chem. 2003;278(8):5513-5516.

44. Anthony RM, Wermeling F, Karlsson MC, Ravetch JV. Identification of a receptor required for the anti-inflammatory activity of IVIG. Proc Natl Acad Sci USA. 2008;105(50):19571-19578.

45. Yokota A, et al. Two species of human Fc epsilon receptor II (Fc epsilon RII/CD23): tissue-specific and IL-4-specific regulation of gene expression. Cell. 1988;55(4):611-618.

46. Delespesse G, Hofstetter H, Sarfati M. Lowaffinity receptor for IgE (FcERII, CD23) and its soluble fragments. Int Arch Allergy Appl Immunol. 1989;90(suppl 1):41-44.

47. Borthakur S, Andrejeva G, McDonnell JM. Basis of the intrinsic flexibility of the $\mathrm{C} \varepsilon 3$ domain of IgE. Biochemistry. 2011;50(21):4608-4614.

48. Dhaliwal B, et al. Crystal structure of IgE bound to its $\mathrm{B}$-cell receptor $\mathrm{CD} 23$ reveals a mechanism of reciprocal allosteric inhibition with high affinity receptor FceRI. Proc Natl Acad Sci U S A. 2012;109(31):12686-12691.

49. Pagan JD, Kitaoka M, Anthony RM. Engineered sialylation of pathogenic antibodies in vivo attenuates autoimmune disease. Cell. 2018;172(3):564-577.e13.

50. Wang TT, et al. IgG antibodies to dengue enhanced for Fc $\gamma$ RIIIA binding determine disease severity. Science. 2017;355(6323):395-398.

51. Kapur R, et al. A prominent lack of IgG1-Fc fucosylation of platelet alloantibodies in pregnancy. Blood. 2014;123(4):471-480.

52. Sonneveld ME, et al. Glycosylation pattern of anti-platelet IgG is stable during pregnancy and predicts clinical outcome in alloimmune thrombocytopenia. Br J Haematol. 2016;174(2):310-320.

53. Wuhrer M, et al. Regulated glycosylation patterns of IgG during alloimmune responses against human platelet antigens. JProteome Res. 2009;8(2):450-456.

54. Thulin NK, Wang TT. The role of Fc gamma receptors in broad protection against influenza viruses. Vaccines (Basel). 2018;6(3):E36.

55. DiLillo DJ, Tan GS, Palese P, Ravetch JV. Broadly neutralizing hemagglutinin stalkspecific antibodies require $\mathrm{Fc} \gamma \mathrm{R}$ interactions for protection against influenza virus in vivo. Nat Med. 2014;20(2):143-151.

56. Marcus R, et al. Obinutuzumab for the first-line treatment of follicular lymphoma. $N$ Engl J Med. 2017;377(14):1331-1344.

57. MacroGenics Announces Positive Results from 
Pivotal Phase 3 SOPHIA Study of Margetuximab [press release]. Globenewswire.com. https://www.globenewswire.com/news-release/2019/02/06/1711231/0/en/MacroGenics-Announces-Positive-Results-from-Pivotal-Phase-3-SOPHIA-Study-of-Margetuximab.html. Published February 6, 2019. Accessed: Aug 8, 2019.

58. DiLillo DJ, Ravetch JV. Differential Fc-receptor engagement drives an anti-tumor vaccinal effect. Cell. 2015;161(5):1035-1045.

59. Seeling M, Brückner C, Nimmerjahn F. Differential antibody glycosylation in autoimmunity: sweet biomarker or modulator of disease activity? Nat Rev Rheumatol. 2017;13(10):621-630.

60. Anthony RM, Nimmerjahn F. The role of differential IgG glycosylation in the interaction of antibodies with Fc $\gamma \mathrm{Rs}$ in vivo. Curr Opin Organ Transplant. 2011;16(1):7-14.

61. Brückner C, Lehmann C, Dudziak D, Nimmerjahn F. Sweet SIGNs: IgG glycosylation leads the way in IVIG-mediated resolution of inflammation. Int Immunol. 2017;29(11):499-509.

62. Selman MH, et al. Changes in antigenspecific IgG1 Fc N-glycosylation upon influenza and tetanus vaccination. Mol Cell Proteomics. 2012;11(4):M111.014563.

63. Jonsson S, et al. Identification of sequence variants influencing immunoglobulin levels. Nat Genet. 2017;49(8):1182-1191.

64. Espéli M, et al. Fc $\gamma$ RIIb differentially regulates pre-immune and germinal center $\mathrm{B}$ cell tolerance in mouse and human. Nat Commun. 2019;10(1):1970.

65. Fukuyama H, Nimmerjahn F, Ravetch JV. The inhibitory Fcgamma receptor modulates autoimmunity by limiting the accumulation of immunoglobulin G+ anti-DNA plasma cells. Nat Immunol. 2005;6(1):99-106.

66. Kono H, et al. FcgammaRIIB Ile232Thr transmembrane polymorphism associated with human systemic lupus erythematosus decreases affinity to lipid rafts and attenuates inhibitory effects on B cell receptor signaling. Hum Mol Genet. 2005;14(19):2881-2892.

67. Li F, Smith P, Ravetch JV. Inhibitory Fcy receptor is required for the maintenance of tolerance through distinct mechanisms. Jimmunol. 2014;192(7):3021-3028

68. McGaha TL, Sorrentino B, Ravetch JV. Restoration of tolerance in lupus by targeted inhibitory receptor expression. Science. 2005;307(5709):590-593.

69. Su K, et al. A promoter haplotype of the immunoreceptor tyrosine-based inhibitory motif-bearing FcgammaRIIb alters receptor expression and associates with autoimmunity. I. Regulatory FCGR2B polymorphisms and their association with systemic lupus erythematosus. JImmunol. 2004;172(11):7186-7191.

70. Tackenberg B, et al. Impaired inhibitory Fcgamma receptor IIB expression on $\mathrm{B}$ cells in chronic inflammatory demyelinating polyneuropathy. Proc Natl Acad Sci U S A. 2009;106(12):4788-4792.

71. Mackay M, et al. Selective dysregulation of the FcgammaIIB receptor on memory B cells in SLE. JExp Med. 2006;203(9):2157-2164.

72. Boruchov AM, Heller G, Veri MC, Bonvini E, Ravetch JV, Young JW. Activating and inhibitory IgG Fc receptors on human DCs mediate opposing functions. JClin Invest. 2005;115(10):2914-2923.

73. Clynes R, Maizes JS, Guinamard R, Ono M, Takai T, Ravetch JV. Modulation of immune complexinduced inflammation in vivo by the coordinate expression of activation and inhibitory Fc receptors. J Exp Med.1999;189(1):179-185.

74. Dhodapkar KM, et al. Selective blockade of inhibitory Fcgamma receptor enables human dendritic cell maturation with IL-12p70 production and immunity to antibody-coated tumor cells. Proc Natl Acad Sci U S A. 2005;102(8):2910-2915.

75. Maamary J, Wang TT, Tan GS, Palese P, Ravetch JV. Increasing the breadth and potency of response to the seasonal influenza virus vaccine by immune complex immunization. Proc Natl Acad Sci U S A. 2017;114(38):10172-10177.

76. Tomana M, Schrohenloher RE, Koopman WJ, Alarcón GS, Paul WA. Abnormal glycosylation of serum IgG from patients with chronic inflammatory diseases. Arthritis Rheum. 1988;31(3):333-338.

77. van de Geijn FE, et al. Immunoglobulin G galactosylation and sialylation are associated with pregnancy-induced improvement of rheumatoid arthritis and the postpartum flare: results from a large prospective cohort study. Arthritis Res Ther. 2009;11(6):R193.

78. Scherer HU, et al. Glycan profiling of anticitrullinated protein antibodies isolated from human serum and synovial fluid. Arthritis Rheum. 2010;62(6):1620-1629.

79. Espy C, et al. Sialylation levels of anti-proteinase 3 antibodies are associated with the activity of granulomatosis with polyangiitis (Wegener's). Arthritis Rheum. 2011;63(7):2105-2115.

80. de Man YA, Dolhain RJ, Hazes JM. Disease activity or remission of rheumatoid arthritis before, during and following pregnancy. Curr Opin Rheumatol. 2014;26(3):329-333.

81. Pfeifle R, et al. Regulation of autoantibody activity by the IL-23- $\mathrm{T}_{\mathrm{H}} 17$ axis determines the onset of autoimmune disease. Nat Immunol. 2017;18(1):104-113.

82. Fokkink WJ, et al. IgG Fc N-glycosylation in Guillain-Barré syndrome treated with immunoglobulins. JProteome Res. 2014;13(3):1722-1730.

83. Engdahl C, et al. Estrogen induces St6gal1 expression and increases Ig $G$ sialylation in mice and patients with rheumatoid arthritis: a potential explanation for the increased risk of rheumatoid arthritis in postmenopausal women. Arthritis Res Ther. 2018;20(1):84.

84. Baković MP, et al. High-throughput IgG Fc $\mathrm{N}$-glycosylation profiling by mass spectrometry of glycopeptides. JProteome Res. 2013;12(2):821-831.

85. Selman MH, et al. Fc specific IgG glycosylation profiling by robust nano-reverse phase HPLCMS using a sheath-flow ESI sprayer interface. JProteomics. 2012;75(4):1318-1329.

86. Wuhrer M, et al. Skewed Fc glycosylation profiles of anti-proteinase 3 immunoglobulin G1 autoantibodies from granulomatosis with polyangiitis patients show low levels of bisection, galactosylation, and sialylation. J Proteome Res. 2015;14(4):1657-1665.

87. Kaneko Y, Nimmerjahn F, Madaio MP, Ravetch JV. Pathology and protection in nephrotoxic nephritis is determined by selective engagement of specific Fc receptors. J Exp Med. 2006;203(3):789-797. 